\title{
The Impact of Years of Schooling on Dementia: Panel Data Evidence from Europe
}

\author{
Ahmet Ozyigit \\ Department of Healthcare Management, University of Mediterranean Karpasia, Nicosia, Cyprus
}

Received July 19, 2020; Revised December 19, 2020; Accepted January 20, 2021

\section{Cite This Paper in the following Citation Styles}

(a): [1] Ahmet Ozyigit , "The Impact of Years of Schooling on Dementia: Panel Data Evidence from Europe," Universal Journal of Public Health, Vol. 9, No. 1, pp. 18-25, 2021. DOI: 10.13189/ujph.2021.090102.

(b): Ahmet Ozyigit (2021). The Impact of Years of Schooling on Dementia: Panel Data Evidence from Europe. Universal Journal of Public Health, 9(1), 18-25. DOI: 10.13189/ujph.2021.090102.

Copyright $\bigcirc 2021$ by authors, all rights reserved. Authors agree that this article remains permanently open access under the terms of the Creative Commons Attribution License 4.0 International License

\begin{abstract}
Dementia is a group of neurodegenerative disorders with multifactorial and complex etiologies. While a specific pathway is not identified, a number of associations have been reported in an attempt to understand the complexity of the condition. There is robust evidence both from cohort and case-control studies regarding the possibly protective effects of years of schooling on developing dementia later on in life. While theories exist why increased educational attainments may protect our cognitive abilities, the relevant literature suffers from a gap in formal empirical cross-country evidence. Recent literature on clinical dementia also suggests a role for cold exposure in development of dementia in the old age, possibly creating a geographic gradient between colder and warmer countries. This study aims to empirically test the relationship between years of schooling and dementia prevalence rates in the 28 EU countries (UK included) with further focus on climate differentials, using panel data estimation. Results from this study provide evidence in favor of a significant negative relationship between years of schooling and dementia prevalence rates in the EU-28. Climate differentials, on the other hand, only provide a weak association. Further research on broader geographic areas can shed more light on this possible association with broader range of temperatures observed.
\end{abstract}

Keywords Dementia, Education, Panel Data, Neurocognitive Disorder, Cold Exposure, Europe

\section{Introduction}

American Psychiatric Association's Diagnostic and
Statistical Manual of Mental Disorders (DSM- V) describe dementia as a major neurocognitive disorder; one which presents with various degrees of acquired cognitive impairment [1]. Dementia is a group of neurodegenerative disorders which includes subtypes such as Alzheimer's disease, cerebrovascular neurocognitive disorder, frontotemporal lobar degeneration, Lewy body dementia to name a few. Age is suggested to be the single most important risk factor for developing neurocognitive disorders [2]. With a continuous global increase in life expectancy from around 35 years in early 1900s to over 70 years in 2019 [3], the global burden of dementia is expected to keep growing significantly without a definitive cure. According to the World Health Organization (WHO), worldwide prevalence of dementia is estimated around 50 million people with an annual incidence of 10 million new cases and a forecast of over 130 million people by the year 2050 [4].

Numerous modifiable and non-modifiable risk factors have been proposed in an attempt to explain differences in incidence across populations and geographical regions. Several studies report an association between midlife hypertension, hypercholesterolemia, and type 2 diabetes (T2D) and dementia later in life [5,6]. On the other hand, education has been proposed as a protective factor against development of neurocognitive disorders with higher educational attainments earlier in life corresponding to lower incidence or burden of dementia in older ages [7,8,9,10,11]. However, a large-scale 2019 publication looks into the relationship between years of schooling and incidence of dementia in a prospective fashion but finds no significant association between incidence or the age of onset of dementia based on educational attainments. The 
average years of schooling of the participants used in the study are given as 16.3 years, suggesting that participants mostly had high levels of schooling to begin with. Therefore, while concluding that they could not find an association, the authors mention that perhaps the association between schooling and dementia could be more pronounced at the lower ends of the spectrum when distinguishing between people with very little to no formal schooling and those with many years of education [12].

A number of cross-country epidemiological studies suggest that countries like Sweden, Canada, the UK, the Netherlands, and the USA are experiencing a decline in the incidence and prevalence of dementia and are expected to continue doing so in the foreseeable future [13]. On the other hand, China, India and Sub-Saharan Africa are showing an increasing trend in dementia prevalence and are expected to show further significant increase over the next 20 years [14]. Apart from geographical locations, these groups of countries differ greatly in their diets, per capita income, access to healthcare and education. Mean years of schooling in the former group of countries average 13 years while this number is about 8 for the latter, not to mention significant male to female difference in the latter [15]. Perhaps not only education but also other social and economic indicators can play a role in such differences, but this paper focuses specifically on education in an attempt to unravel a possible relationship at an empirical level.

While country-specific and regional micro-scale cohort and case-control studies exist, this paper uses aggregate level panel data evidence from Europe to empirically test whether countries with higher levels of schooling in fact experience lower prevalence of dementia. Local clinical studies or even multi-center studies can only focus on a limited number of people who actively participate in such studies. Furthermore, these studies look at educational attainments as well as many other parameters and their association with developing dementia later on in life on an individual basis either prospectively or retrospectively. Even though these studies contribute significantly to our understanding of how dementia prevalence correlates with certain parameters, they cannot account for regional or cross-country differences. From an education point of view, heterogeneities can exist across countries as well as within countries with respect to the educational system, culture of education, quality of education or many other possible influences that can make years of schooling a non-standard construct. The model introduced in this paper takes into account both cross-country and cross-time differentials that can be expected across countries. In other words, the model is able to account for heterogeneities across countries and isolate the effect of years of schooling from other country-specific elements for a more accurate depiction of the relationship between years of schooling and dementia prevalence.
Apart from the effects of years of schooling on dementia prevalence, this paper also attempts to address a second research question. Based on clinical studies, cold exposure has been shown to have an association with neurodegeneration. Research shows that older people tend to express lower body temperatures compared to the younger population, making them susceptible to metabolic problems that may be associated with defective thermoregulation [16,17]. Moreover, in-vivo animal studies show a clear link between hypothermia and tau protein hyperphosphorylation, which tends to be the main pathophysiological event in various subtypes of dementias $[18,19]$. If these findings are generalizable to larger populations, one could possibly argue that cold climates can contribute to the development of dementia. For this end, cold exposure is used as an additional explanatory variable when exploring differences in dementia prevalence across Europe.

The rest of the paper is structured as follows: Section 2 presents the relevant literature; section 3 acquaints the reader with the data and model selection; section 4 presents and discusses the main findings and finally, section 5 concludes with an overview of future directions of research.

\section{Literature Review}

Lower levels of formal education have been found to be associated with higher likelihood of dementia in later stages of life. Possible explanations for this relationship focus on insufficient brain stimulation at critical developmental periods as well as the likelihood of unhealthier lifestyle choices resulting in exposure to more risk factors, suggesting that education is not only a direct way of improving one's cognitive abilities, but also an indirect means of cultivating a higher quality of life via well-informed lifestyle choices. An even more striking argument has also gained popularity, suggesting that those who are highly educated tend to develop higher brain function that can better compensate for the neurocognitive burden induced by dementia [20,21,22].

Literature provides evidence for a positive impact on diet and exercise habits as a result of increased years of schooling $[23,24,25,26]$. Higher educational attainments do not only raise individuals' awareness of healthy life choices but also increase their likelihood of securing higher paid jobs, allowing them access to higher quality of food and living standards. Adults with lower years of education report poorer overall health $[27,28]$ as well as higher incidence of chronic conditions [29,30]. Hahn \& Truman observe a dose-response relationship between years of schooling and various health behavior such as cigarette smoking, alcohol consumption and physical activity among the adult population in the United States [31]. Such behaviors are strongly associated with conditions such as hypertension, hypercholesterolemia 
and T2D, which, incidentally, are among the major modifiable risk factors suggested for dementia.

Larsson et al. find that each additional year of formal education appears to be associated with a lower risk of developing dementia later in life ( odds ratio [OR] 0.89 ; 95\% confidence interval [CI] 0.84 to 0.93) [8]. Authors also provide evidence for a significant reduction of dementia risk among university graduates. Meng and D'Arcy, in their 2012 meta- analysis, find a statistically significant odds ratio evidence 2.61 (95\% CI 2.21-3.07) for prevalence studies and 1.88 (95\% CI 1.51-2.34) for incidence studies for the relationship between lower level of schooling and burden of dementia of all sub-types [32]. Similarly, Brayne et al. provide evidence for an education gradient with more exposure to formal education leading to a lower risk of dementia at the time of death (odds ratio [OR] 0.89; 95\% confidence interval [CI] 0.83 to 0.94) [7]. With ample evidence suggesting improved health behavior and outcomes as a response to increased levels of education, one could argue that improved level of schooling could act as a proxy for better lifestyle choices that can possibly reduce the exposure to risk factors which can contribute or worsen the outcomes of chronic conditions including neurocognitive disorders.

Old age has always been associated with neurodegenerative disorders, suggesting that aging is responsible for changes in the brain that can render the elderly vulnerable for developing neurodegeneration. However, even though age is considered to be the most important factor, not all people in the old age develop dementia. This raises the question whether there are other predisposing factors that can expedite or amplify the effects of old age. Recently, defects in thermoregulation have been implicated as a possible cause of neurodegenerative pathologies in the brain. In a 2016 laboratory study, mice with artificially induced Alzheimer's disease were found to exhibit deficits in thermoregulation, reducing their core body temperatures [33]. Moreover, in a more recent laboratory study in 2019, mice that have been exposed to colder temperatures were found to have increased tau phosphorylation- a pathophysiological marker for worsening neurodegeneration [34]. What these recent studies indicate is that cold exposure can possibly contribute to development of neurodegenerative diseases. Furthermore, once neurodegeneration develops, it may further induce thermoregulatory deficits, almost creating a vicious cycle. This paper aims to empirically test the possible relationship between climate differentials across Europe and dementia prevalence in an attempt to understand whether colder climates put the elderly at an elevated risk of developing dementia in general.

\section{Materials and Methods}

The survey used in this paper is based on dementia prevalence rates obtained from the Institute for Health
Metrics and Evaluation (IHME) 2017 publication covering the period between 1990-2017 [35]. Data from all $28 \mathrm{EU}$ states have been incorporated into this study (UK is also included in the data set). The countries have been classified into "colder" and "warmer" based on winter extreme temperatures as a proxy for cold exposure. Country based education statistics come from Barro-Lee and Human Development Reports [36,37], and cover the period of 1990-2017 as well. Post-2017 data are not yet available for dementia statistics; therefore, we use data until 2017 for both variables for a balanced panel dataset. The following variables have been used in the model:

DemPrev represents the annual dementia prevalence for the 28-member states covering the period between 1990-2017. The values are used in the model after logarithmic transformation in order to reduce variability between observations and normalize the distribution.

Edu represents logarithmic transformation of the mean years of schooling for each member state for the same period.

Cold is a dummy variable used to distinguish between the member states that have more exposure to extreme weathers in winter. A value of 1 has been assigned to countries with an average winter temperature of below zero. A value of 0 has been assigned to others.

When using panel data analysis, OLS estimation with pooled panel appears to be a relatively simple approach. However, this approach has an embedded assumption of no unique attributes between the cross-sectional entities (The EU member states). This is quite a restrictive assumption considering demographic, environmental, ethnic and cultural differences across the populations of the EU member states. As a result, this paper will consider fixed and random effects models which take into consideration cross-country heterogeneities. The two models have their own strengths/weaknesses depending on the type of data and measurement being considered. In order to decide between the two, Wu Haussmann test has been employed. Based on the test results, the null hypothesis of random effects cannot be rejected. Therefore, the random effects model appears to be more suitable for the current paper [38].

This study estimates two models in an attempt to explain the effect of years of schooling on dementia prevalence in the $28 \mathrm{EU}$ member states. A dummy variable also enters the equation in an attempt to isolate the possible effect of cold exposure on dementia. These models are as follows:

$$
\begin{gathered}
\text { DemPrev }=f(\text { Edu }) \\
\text { DemPrev }=f(\text { Edu, Cold })
\end{gathered}
$$

In the first equation, we attempt to explain dementia prevalence as a function of years of schooling. A negative coefficient on years of schooling will imply a protective effect of education against dementia. In the second equation, a second explanatory variable has been added to 
the relationship. In this equation, dementia prevalence rate is explained by years of schooling and cold exposure. If cold weather has any influence on developing dementia, then we expect a positive and statistically significant coefficient for the cold variable.

While the model proposed in this study aims to measure the association between education, cold exposure and dementia prevalence, it is far from giving a complete picture of how dementia comes about. However, when dealing with complex neurological phenomena such as dementia and neurodegenerative diseases, even the smallest findings can form the basis for more advanced findings.

One of the shortcomings of this paper is that temperature differentials across Europe do not provide too wide of a range. In further studies, more geographical regions with higher temperature differentials should be used to assess the role of cold exposure on a wider level.

\section{Results and Discussion}

Table 1 below provides the estimation output for the three models specified in this paper.

Table 1. Panel Data Estimates

\begin{tabular}{|c|c|c|c|c|}
\hline & $\begin{array}{c}\text { Pooled } \\
\text { Panel }\end{array}$ & $\begin{array}{c}\text { Pooled } \\
\text { Panel }\end{array}$ & $\begin{array}{c}\text { Random } \\
\text { Effects }\end{array}$ & $\begin{array}{l}\text { Random } \\
\text { Effects }\end{array}$ \\
\hline Variable & $\begin{array}{l}\text { Demprev } \\
=\mathrm{f}(\text { Edu })\end{array}$ & $\begin{array}{l}\text { Demprev } \\
=\mathrm{f}(\text { Edu, } \\
\text { Cold })\end{array}$ & $\begin{array}{l}\text { Demprev } \\
=\mathrm{f}(\text { Edu })\end{array}$ & $\begin{array}{l}\text { Demprev } \\
=\mathrm{f}(\text { Edu, } \\
\text { Cold })\end{array}$ \\
\hline Constant & $6.80 * * *$ & $6.83 * * *$ & $6.67 * * *$ & $6.93 * * *$ \\
\hline Edu & $\begin{array}{l}-0.16 \\
* * *\end{array}$ & $-0.17 * * *$ & $-0.22 * * *$ & $-0.22 * * *$ \\
\hline Cold & & $0.02 * * *$ & & 0.02 \\
\hline $\mathrm{R}^{2}$ & 0.12 & 0.14 & 0.48 & 0.49 \\
\hline
\end{tabular}

Note: ${ }^{*}, * *$ and $* * *$ denote significance at $10 \%, 5 \%$ and $1 \%$, respectively.

Through its direct effects on cognitive function and indirect effects on better lifestyle choices, education appears to have significance beyond its face value when used as an explanatory variable in health outcome measurements. In both pooled panel data estimates and the random effects models, we observe a statistically significant negative coefficient for the years of education when used as an explanatory variable for dementia prevalence, as evidenced by statistically significant negative coefficients. In the pooled panel estimates, a coefficient of -0.17 is estimated for the education variable. According to this estimate, one-year increase in years of schooling is expected to yield a 17 percent decrease in dementia prevalence rates. In other words, each additional year of schooling is expected to have a protective effect against developing dementia later on in life. The random effect estimate, which considers heterogeneities between countries across demographics and time, finds an even higher association between years of schooling and dementia prevalence after accounting for these heterogeneities. According to this estimation output, a one-year increase in years of schooling is expected to reduce dementia prevalence rates by 22 percent.

In pooled panel estimates, cold dummy appears as a significant explanatory variable for dementia prevalence with a small positive coefficient. A coefficient of 0.02 suggests that countries that have an average daily temperature of zero Celsius or lower tend to have, on average, two percent higher prevalence of dementia compared to warmer countries. However, the significance is lost in the random effects model although the sign is still positive. This may suggest that there might be other heterogeneities between the countries in this model that can better explain the varying dementia prevalence rates. It may also be the case that a single dummy for cold exposure may not be sufficient to unravel the relationship and perhaps the model can further be developed by incorporating a variable in order to measure the impact of specific temperatures on dementia prevalence rates.

The goodness of fit, as measured by $\mathrm{R}^{2}$, appears to improve significantly in the random effects model. A slight improvement can also be observed when the cold dummy is introduced in both models. An $\mathrm{R}^{2}$ close to one is usually accepted as a model having evidence of sufficient explanatory power. However, we would not expect to see such powerful explanatory power of $\mathrm{R}^{2}$ in this model as it would be difficult to explain such a complex medical condition with only a few variables.

Figure 1 displays the scatter plot diagrams for years of education and dementia prevalence rates in the EU-28 for a visual representation of the relationship between the two variables: 

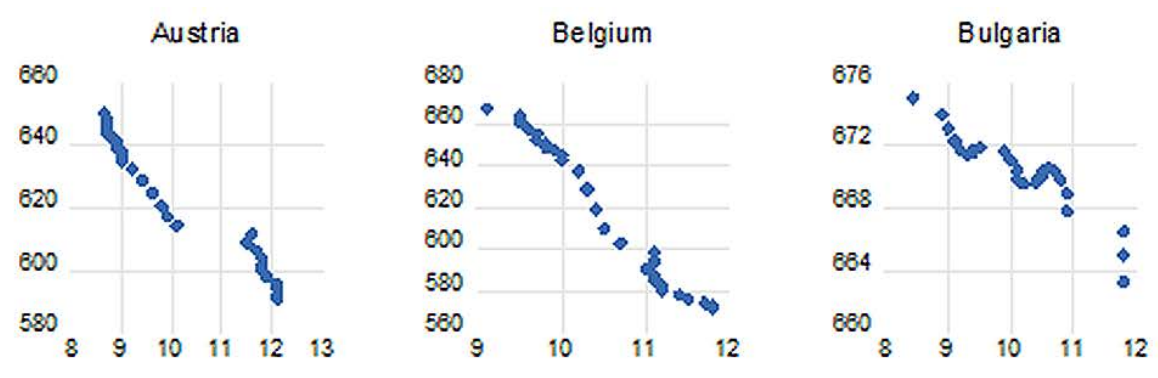

Denmark

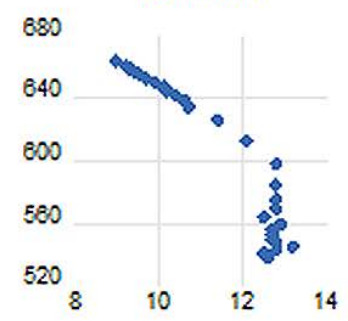

\section{Estonia}
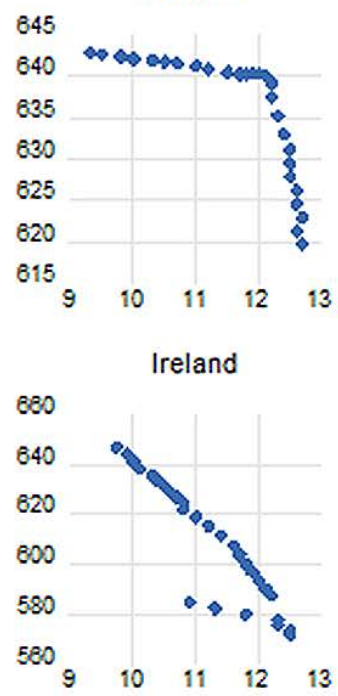

$$
\text { Malta }
$$

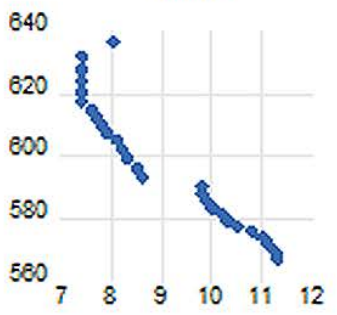

Slovenia

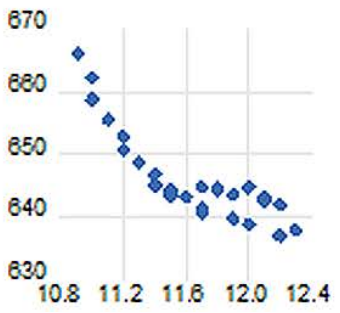

Netherlands

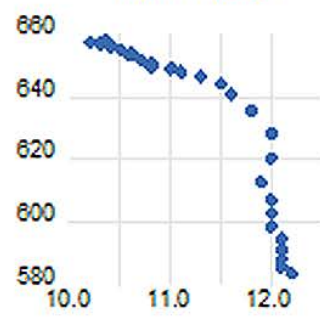

Spain

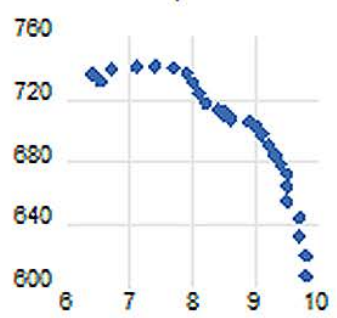

Finland

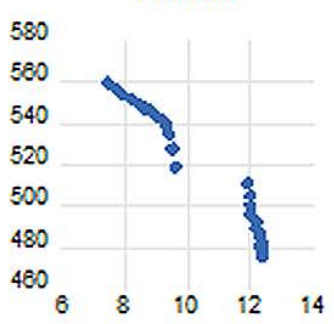

Italy

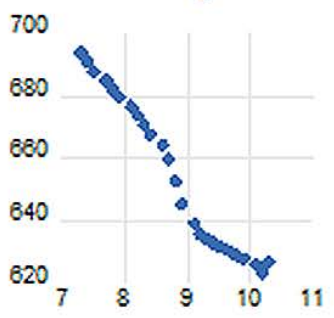

Poland

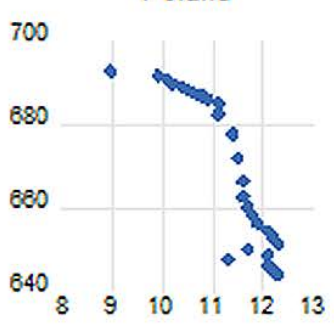

Sweden

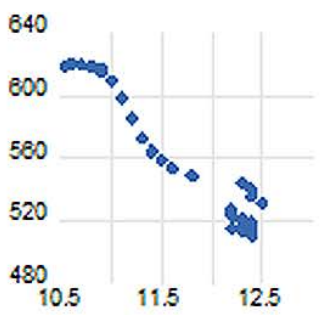

Figure 1. Scatter Plot Diagram of the Relationship between Years of Schooling (Y-axis) and Dementia Prevalence (X-axis) in the EU-28 Countries. 

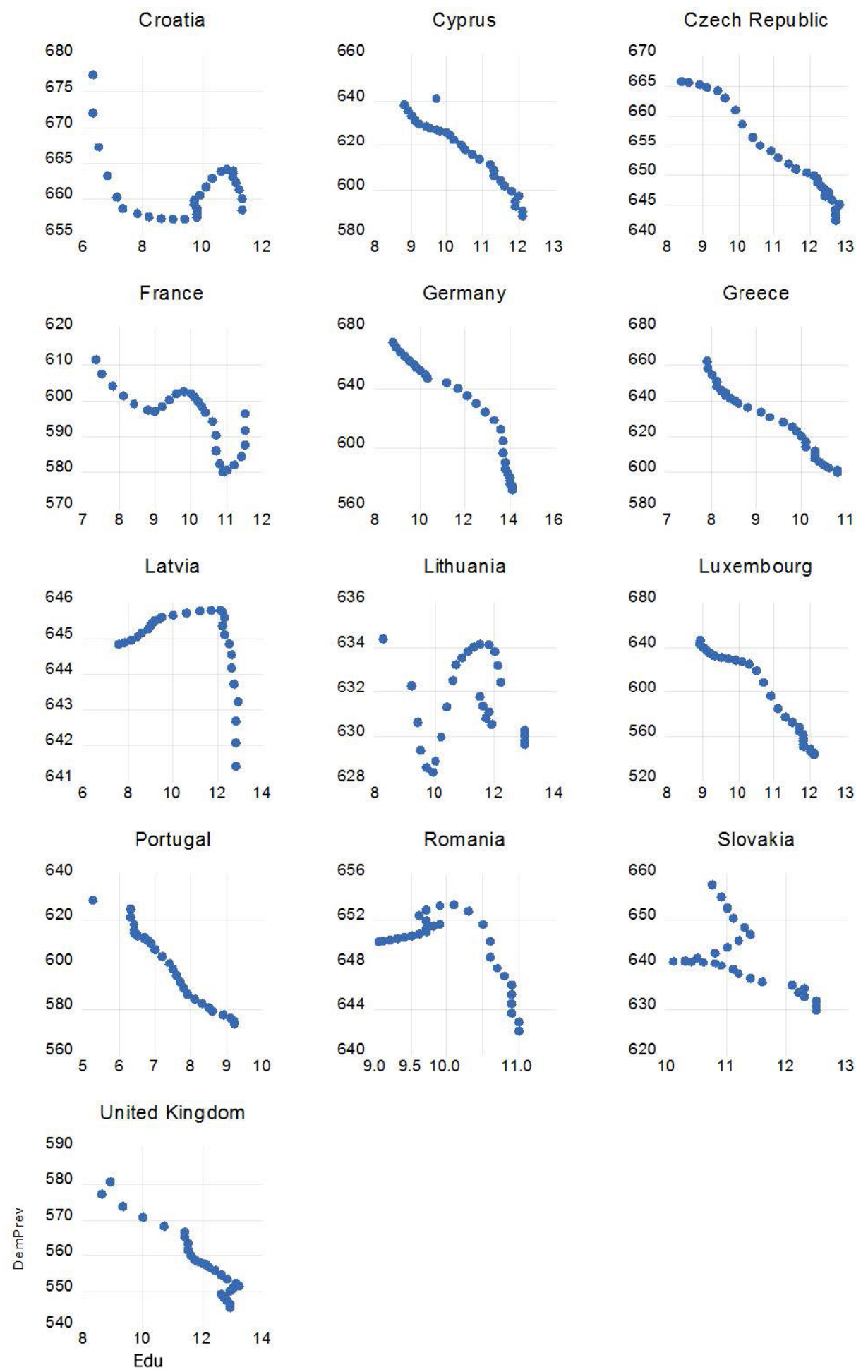

Figure 1 (cont'd). Scatter Plot Diagram of the Relationship between Years of Schooling (Y-axis) and Dementia Prevalence (X-axis) in the EU-28 Countries.

Even though some of the countries do not fit the exact pattern, we can see that majority of the countries exhibit a linear negative relationship between education and dementia prevalence, which further strengthens the estimation output from the empirical model.

While cold exposure appears to have a weak association, if any, with dementia prevalence rates, years of education has a highly significant negative association with dementia prevalence in EU-28 countries. Therefore, this article succeeds in providing, for the first time in literature, formal empirical evidence for a negative association between years of education and dementia prevalence rates in the EU countries via cross-sectional comparison. Moreover, the statistically significant cold exposure 
dummy in the pooled panel estimates is worth a closer analysis in subsequent studies, perhaps with further considerations.

\section{Conclusions}

There is clear evidence both from cohort and case-control studies regarding the possibly protective effects of years of schooling on developing dementia later on in life. Due to the nature of these studies, a long-term follow-up of individuals is required. This requires a considerable amount of time and resources, limiting the availability of such studies at larger scales. Even if larger numbers of people are studied, isolating cross-country heterogeneities is not possible. While the current study is far from depicting a perfect picture, it contributes to our understanding of the protective effects of years of formal schooling on dementia prevalence rates by also accounting for other possible cross-country heterogeneities using econometric analysis. The results show a significant protective effect of years of schooling on developing dementia later on in life. This is quantified as a 17 percent reduction in dementia prevalence as a response to a one-year increase in schooling. Even though dementia cannot be explained by a single variable, being able to isolate the effect of education from possible confounders provides a solid finding for the protective role of education on neurodegeneration.

Recent literature on clinical dementia studies also suggests a role for cold exposure in development of dementia in the old age, possibly creating a gradient between the colder and warmer climates. In current work, cold exposure has been introduced as a dummy variable to categorize the EU-28 countries into two groups according to their climates. While cold exposure appears to have a positive association with dementia prevalence rates in pooled panel estimates, the significance of its impact is not observed when other cross-country heterogeneities are figured into the model. Further studies with inclusion of broader geographic regions with higher temperature differentials can possibly shed more light into the specific impact of air temperatures in developing dementia. However, based on the results of this article, it is not possible to say that cold exposure contributes to dementia.

\section{Funding}

This research received no external funding.

\section{Conflict of Interest}

Author declares no conflict of interest.

\section{REFERENCES}

[1] Guerreiro, Rita, and Jose Bras. "The Age Factor in Alzheimer’s Disease". Genome Medicine, vol 7, no. 1, 2015. Springer Science And Business Media LLC, DOI:10.1186/s 13073-015-0232-5.

[2] Roser, Max et al. "Life Expectancy". Our World In Data, 2020, https://ourworldindata.org/life-expectancy.

[3] Prince, Martin et al. World Alzheimer Report 2015: The Global Impact of Dementia. Alzheimers Disease International (ADI), London, 2015, http://www.worldalzrep ort2015.org/downloads/world-alzheimer-report-2015.pdf. Accessed 14 Apr 2020.

[4] Kivipelto, Miia et al. "Obesity and Vascular Risk Factors At Midlife And The Risk Of Dementia And Alzheimer Disease". Archives of Neurology, vol 62, no. 10, 2005. American Medical Association (AMA), DOI:10.1001/archne ur.62.10.1556.

[5] Schnaider Beeri, M. et al. "Diabetes Mellitus In Midlife And The Risk Of Dementia Three Decades Later". Neurology, vol 63, no. 10, 2004, pp. 1902-1907. Ovid Technologies (Wolters Kluwer Health), DOI:10.1212/01.wnl.0000144278 .79488.dd.

[6] Brayne, Carol et al. "Education, The Brain And Dementia: Neuroprotection Or Compensation?". Brain, vol 133, no. 8, 2010, pp. 2210-2216. Oxford University Press (OUP), DOI:10.1093/brain/awq185.

[7] Larsson, Susanna C et al. "Modifiable Pathways In Alzheimer's Disease: Mendelian Randomisation Analysis". BMJ, 2017, p. j5375. BMJ, DOI:10.1136/bmj.j5375.

[8] Ngandu, T. et al. "Education And Dementia: What Lies Behind The Association?". Neurology, vol 69, no. 14, 2007, pp. 1442-1450. Ovid Technologies (Wolters Kluwer Health), doi:10.1212/01.wnl.0000277456.29440.16.

[9] Oghagbon, Efosa Kenneth, and Lydia Giménez - Llort. "Short Height And Poor Education Increase The Risk Of Dementia In Nigerian Type 2 Diabetic Women". Alzheimer's \& Dementia: Diagnosis, Assessment \& Disease Monitoring, vol 11, no. 1, 2019, pp. 493-499. Wiley, doi:10.1016/j.dadm 2019.05.006.

[10] Paddick, Stella-Maria et al. "The Association between Educational Level and Dementia In Rural Tanzania". Dementia \& Neuropsychologia, vol 8, no. 2, 2014, pp. 117-125. Fapunifesp (Scielo), DOI:10.1590/s1980-5764201 4dn82000006.

[11] Wilson, Robert S. et al. "Education And Cognitive Reserve In Old Age". Neurology, vol 92, no. 10, 2019, pp. e1041-e1050. Ovid Technologies (Wolters Kluwer Health), DOI:10.1212/wnl.0000000000007036.

[12] Matthews, Fiona E et al. "A Two-Decade Comparison of Prevalence of Dementia in Individuals Aged 65 Years And Older From Three Geographical Areas Of England: Results Of The Cognitive Function And Ageing Study I And II". The Lancet, vol 382, no. 9902, 2013, pp. 1405-1412. Elsevier BV, DOI:10.1016/s0140-6736(13)61570-6.

[13] Prince, Martin et al. "The Global Prevalence Of Dementia: A Systematic Review And Meta-analysis". Alzheimer's \& Dementia, vol 9, no. 1, 2013, pp. 63-75.e2. Wiley, 
DOI:10.1016/j.jalz.2012.11.007.

[14] UNDP. "Human Development Reports". Hdr.Undp.Org, 2020, http://hdr.undp.org/en/indicators/103006.

[15] Kelly, G. Body temperature variability (Part 1): a review of the history of body temperature and its variability due to site selection, biological rhythms, fitness, and aging. Alternative Medicine Review, vol. 11, 2006, pp278-93.

[16] Waalen, J., and J. N. Buxbaum. "Is Older Colder or Colder Older? The Association of Age with Body Temperature in 18,630 Individuals". The Journals of Gerontology Series A: Biological Sciences and Medical Sciences, vol. 66, no. 5, 2011, pp. 487-492. Oxford University Press (OUP), DOI:10.1093/gerona/glr001.

[17] Carrettiero, Daniel Carneiro et al. "Temperature and Toxic Tau In Alzheimer's Disease: New Insights". Temperature, vol 2, no. 4, 2015, pp. 491-498. Informa UK Limited, DOI:10.1080/23328940.2015.1096438.

[18] Le Freche, Hélène et al. "Tau Phosphorylation and Sevoflurane Anesthesia". Anesthesiology, vol 116, no. 4, 2012, pp. 779-787. Ovid Technologies (Wolters Kluwer Health), DOI:10.1097/aln.0b013e31824be8c7.

[19] Stern, Yaakov. "Cognitive Reserve and Alzheimer Disease". Alzheimer Disease \& Associated Disorders, vol 20, no. Supplement 2, 2006, pp. S69-S74. Ovid Technologies (Wolters Kluwer Health), DOI:10.1097/00002093-2006070 01-00010. Accessed 17 Sep 2020.

[20] Valenzuela, Michael J., and Perminder Sachdev. "Brain Reserve and Cognitive Decline: A Non-Parametric Systematic Review". Psychological Medicine, vol 36, no. 8, 2006, pp. 1065-1073. Cambridge University Press (CUP), DOI:10.1017/s0033291706007744. Accessed 19 Sep 2020.

[21] Valenzuela, Michael J. "Brain Reserve and the Prevention of Dementia". Current Opinion In Psychiatry, vol. 21, no. 3, 2008, pp. 296-302. Ovid Technologies (Wolters Kluwer Health), DOI:10.1097/yco.0b013e3282f97b1f. Accessed 11 Aug 2020.

[22] Droomers, Mariël et al. "Educational Differences in Excessive Alcohol Consumption: The Role of Psychosocial and Material Stressors". Preventive Medicine, vol 29, no. 1, 1999, pp. 1-10. Elsevier BV, DOI:10.1006/pmed.1999.0496.

[23] Cutler, David M., and Edward L. Glaeser. "What Explains Differences in Smoking, Drinking And Other Health-Related Behaviors?". SSRN Electronic Journal, 2005. Elsevier BV, DOI:10.2139/ssrn.658302. Accessed 03 Mar 2020.

[24] Cutler, David M., and Adriana Lleras-Muney. "Understanding Differences in Health Behaviors By Education". Journal of Health Economics, vol 29, no. 1, 2010, pp. 1-28. Elsevier BV, DOI:10.1016/j.jhealeco.2009.1 0.003 .

[25] Li, Jinhu, and Nattavudh Powdthavee. "Does More Education Lead To Better Health Habits? Evidence from The School Reforms In Australia". Social Science \& Medicine, vol. 127, 2015, pp. 83-91. Elsevier BV,
DOI:10.1016/j.socscimed.2014.07.021.

[26] Mirowsky, John, and Catherine E. Ross. "Education and Self-Rated Health". Research on Aging, vol 30, no. 1, 2008, pp. 93-122. SAGE Publications, DOI:10.1177/0164027507 309649.

[27] Zajacova, Anna et al. "Education and Health Among U.S. Working-Age Adults: A Detailed Portrait Across The Full Educational Attainment Spectrum". Biodemography and Social Biology, vol 58, no. 1, 2012, pp. 40-61. Informa UK Limited, DOI:10.1080/19485565.2012.666122.

[28] Johnson-Lawrence, Vicki et al. "Education, Race/Ethnicity, and Multimorbidity Among Adults Aged 30-64 In The National Health Interview Survey". SSM - Population Health, vol 3, 2017, pp. 366-372. Elsevier BV, DOI:10.1016/j.ssmph.2017.03.007.

[29] Quiñones, Ana R. et al. "Multimorbidity Combinations and Disability in Older Adults". The Journals of Gerontology Series A: Biological Sciences and Medical Sciences, vol 71, no. 6, 2016, pp. 823-830. Oxford University Press (OUP), DOI:10.1093/gerona/glw035.

[30] Hahn, Robert A., and Benedict I. Truman. "Education Improves Public Health and Promotes Health Equity". International Journal of Health Services, vol 45, no. 4, 2015, pp. 657-678. SAGE Publications, DOI:10.1177/002073141 5585986 .

[31] Meng, Xiangfei, and Carl D’Arcy. "Education and Dementia in The Context of The Cognitive Reserve Hypothesis: A Systematic Review With Meta-Analyses And Qualitative Analyses". Plos ONE, vol 7, no. 6, 2012, p. e38268. Public Library Of Science (Plos), DOI:10.1371/journal.pone.0038 268.

[32] Vandal, Milene et al. "Impaired Thermoregulation and Beneficial Effects of Thermoneutrality in The $3 \times \mathrm{Tg}-\mathrm{AD}$ Model Of Alzheimer's Disease". Neurobiology of Aging, vol 43, 2016, pp. 47-57. Elsevier BV, DOI:10.1016/j.neurobiola ging.2016.03.024.

[33] Tournissac, Marine et al. "Repeated Cold Exposures Protect a Mouse Model of Alzheimer's Disease Against Cold-Induced Tau Phosphorylation". Molecular Metabolism, vol 22, 2019, pp. 110-120. Elsevier BV, DOI:10.1016/j.molmet.2019.01.008.

[34] Global Burden of Disease Collaborative Network. Global Burden of Disease Study 2016 (GBD 2016) Cause-Specific Mortality 1980-2016. Seattle, United States: Institute for Health Metrics and Evaluation (IHME), 2017.

[35] Barro, Robert J., and Jong Wha Lee. "A New Data Set of Educational Attainment in The World, 1950-2010". Journal of Development Economics, vol 104, 2013, pp. 184-198. Elsevier BV, DOI:10.1016/j.jdeveco.2012.10.001.

[36] UNDP, "Human Development Reports". Human Development Data Center, 2020, http://hdr.undp.org/en/data. Accessed 11 June 2020.

[37] Greene, William H. Econometric Analysis. 6th ed., Prentice Hall, 2008. 